\title{
Complete Moment Convergence for Arrays of Rowwise $\varphi$-Mixing Random Variables
}

\author{
Lulu Zheng, Yanfang Liu, and Xuejun Wang \\ School of Mathematical Science, Anhui University, Hefei 230601, China \\ Correspondence should be addressed to Xuejun Wang; wxjahdx2000@126.com
}

Received 20 February 2014; Accepted 28 April 2014; Published 11 May 2014

Academic Editor: Ming Mei

Copyright ( 2014 Lulu Zheng et al. This is an open access article distributed under the Creative Commons Attribution License, which permits unrestricted use, distribution, and reproduction in any medium, provided the original work is properly cited.

We investigate the complete moment convergence for maximal partial sum of arrays of rowwise $\varphi$-mixing random variables under some more general conditions. The results obtained in the paper generalize and improve some known ones.

\section{Introduction}

Let $\left\{X_{n}, n \geq 1\right\}$ be a sequence of random variables defined on a fixed probability space $(\Omega, \mathscr{F}, P)$. Let $n$ and $m$ be positive integers. Write $\mathscr{F}_{n}^{m}=\sigma\left(X_{i}, n \leq i \leq m\right)$. Given $\sigma$-algebras $\mathscr{B}, \mathscr{R}$ in $\mathscr{F}$, let

$$
\varphi(\mathscr{B}, \mathscr{R})=\sup _{A \in \mathscr{B}, B \in \mathscr{R}, P(A)>0}|P(B \mid A)-P(B)| .
$$

Define the $\varphi$-mixing coefficients by

$$
\varphi(n)=\sup _{k \geq 1} \varphi\left(\mathscr{F}_{1}^{k}, \mathscr{F}_{k+n}^{\infty}\right), \quad n \geq 0 .
$$

A random variable sequence $\left\{X_{n}, n \geq 1\right\}$ is said to be $\varphi$-mixing if $\varphi(n) \downarrow 0$ as $n \rightarrow \infty . \varphi(n)$ is called mixing coefficient. A triangular array of random variables $\left\{X_{n k}, k \geq 1, n \geq 1\right\}$ is said to be an array of rowwise $\varphi$ mixing random variables if, for every $n \geq 1,\left\{X_{n k}, k \geq 1\right\}$ is a $\varphi$-mixing sequence of random variables. The notion of $\varphi$ mixing random variables was introduced by Dobrushin [1] and many applications have been found. See, for example, Utev [2] for central limit theorem, Gan and Chen [3] for limit theorem, Peligrad [4] for weak invariance principle, Shao [5] for almost sure invariance principles, Chen and Wang [6], Shen et al. [7, 8], Wu [9], and Wang et al. [10] for complete convergence, $\mathrm{Hu}$ and Wang [11] for large deviations, and so forth. When these are compared with corresponding results of independent random variable sequences, there still remains much to be desired.
Definition 1. A sequence of random variables $\left\{U_{n}, n \geq 1\right\}$ is said to converge completely to a constant $a$ if, for any $\varepsilon>0$,

$$
\sum_{n=1}^{\infty} P\left(\left|U_{n}-a\right|>\varepsilon\right)<\infty
$$

In this case, one writes $U_{n} \rightarrow a$ completely. This notion was given first by Hsu and Robbins [12].

Definition 2. Let $\left\{Z_{n}, n \geq 1\right\}$ be a sequence of random variables and $a_{n}>0, b_{n}>0$, and $q>0$. If

$$
\sum_{n=1}^{\infty} a_{n} E\left\{b_{n}^{-1}\left|Z_{n}\right|-\varepsilon\right\}_{+}^{q}<\infty \quad \forall \varepsilon>0
$$

then the above result was called the complete moment convergence by Chow [13].

Let $\left\{X_{n k}, k \geq 1, n \geq 1\right\}$ be an array of rowwise $\varphi$-mixing random variables with mixing coefficients $\{\varphi(n), n \geq 1\}$ in each row, let $\left\{a_{n}, n \geq 1\right\}$ be a sequence of positive real numbers such that $a_{n} \uparrow \infty$, and let $\left\{\Psi_{k}(t), k \geq 1\right\}$ be a sequence of positive even functions such that

$$
\frac{\Psi_{k}(|t|)}{|t|^{q}} \uparrow, \quad \frac{\Psi_{k}(|t|)}{|t|^{p}} \downarrow \quad \text { as }|t| \uparrow
$$


for some $1 \leq q<p$ and each $k \geq 1$. In order to prove our results, we mention the following conditions:

$$
\begin{gathered}
E X_{n k}=0, \quad k \geq 1, n \geq 1, \\
\sum_{n=1}^{\infty} \sum_{k=1}^{n} E \frac{\Psi_{k}\left(X_{n k}\right)}{\Psi_{k}\left(a_{n}\right)}<\infty, \\
\sum_{n=1}^{\infty}\left(\sum_{k=1}^{n} E\left(\frac{X_{n k}}{a_{n}}\right)^{2}\right)^{v / 2}<\infty,
\end{gathered}
$$

where $v \geq p$ is a positive integer.

The following are examples of function $\Psi_{k}(t)$ satisfying assumption (5): $\Psi_{k}(t)=|t|^{\beta}$ for some $q<\beta<p$ or $\Psi_{k}(t)=|t|^{q} \log \left(1+|t|^{p-q}\right)$ for $t \in(-\infty,+\infty)$. Note that these functions are nonmonotone on $t \in(-\infty,+\infty)$, while it is simple to show that, under condition (5), the function $\Psi_{k}(t)$ is an increasing function for $t>0$. In fact, $\Psi_{k}(t)=$ $\left(\Psi_{k}(t) /|t|^{q}\right) \cdot|t|^{q}, t>0$, and $|t|^{q} \uparrow$ as $|t| \uparrow$; then we have $\Psi_{k}(t) \uparrow$.

Recently Gan et al. [14] obtained the following complete convergence for $\varphi$-mixing random variables.

Theorem A. Let $\left\{X_{n}, n \geq 1\right\}$ be a sequence of $\varphi$-mixing mean zero random variables with $\sum_{n=1}^{\infty} \varphi^{1 / 2}(n)<\infty$, let $\left\{a_{n}, n \geq 1\right\}$ be a sequence of positive real numbers with $a_{n} \uparrow \infty$, and let $\left\{\Psi_{n}(t), n \geq 1\right\}$ be a sequence of nonnegative even functions such that $\Psi_{n}(t)>0$ as $t>0$ and $\left(\Psi_{n}(|t|) /|t|\right) \uparrow$ and $\left(\Psi_{n}(|t|) /|t|^{p}\right) \downarrow$ as $|t| \uparrow \infty$, where $p \geq 2$. If the following conditions are satisfied:

$$
\begin{aligned}
& \sum_{n=1}^{\infty} \sum_{k=1}^{n} E \frac{\Psi_{k}\left(X_{k}\right)}{\Psi_{k}\left(a_{n}\right)}<\infty, \\
& \sum_{n=1}^{\infty}\left[\sum_{k=1}^{n} \frac{E\left|X_{k}\right|^{r}}{a_{n}^{r}}\right]^{s}<\infty,
\end{aligned}
$$

where $0<r \leq 2, s>0$, then

$$
\frac{1}{a_{n}} \max _{1 \leq j \leq n}\left|\sum_{k=1}^{j} X_{k}\right| \longrightarrow 0 \quad \text { completely. }
$$

For more details about this type of complete convergence, one can refer to Gan and Chen [3], Wu et al. [15], Wu [16], Huang et al. [17], Shen [18], Shen et al. [19, 20], and so on. The purpose of this paper is extending Theorem A to the complete moment convergence, which is a more general version of the complete convergence, and making some improvements such that the conditions are more general. In this work, the symbol $C$ always stands for a generic positive constant, which may vary from one place to another.

\section{Preliminary Lemmas}

In this section, we give the following lemma which will be used to prove our main results.

Lemma 3 (cf. Wang et al. [10]). Let $\left\{X_{n}, n \geq 1\right\}$ be a sequence of $\varphi$-mixing random variables satisfying $\sum_{n=1}^{\infty} \varphi^{1 / 2}(n)<\infty$, $p \geq 2$. Assume that $E X_{n}=0$, and $E\left|X_{n}\right|^{p}<\infty$, for each $n \geq 1$.
Then there exists a constant $C$ depending only on $p$ and $\varphi(\cdot)$ such that

$$
E\left(\max _{1 \leq j \leq n}\left|\sum_{i=a+1}^{a+j} X_{i}\right|^{p}\right) \leq C\left[\sum_{i=a+1}^{a+n} E\left|X_{i}\right|^{p}+\left(\sum_{i=a+1}^{a+n} E X_{i}^{2}\right)^{p / 2}\right],
$$

for every $a \geq 0$ and $n \geq 1$. In particular, one has

$$
E\left(\max _{1 \leq j \leq n}\left|\sum_{i=1}^{j} X_{i}\right|^{p}\right) \leq C\left[\sum_{i=1}^{n} E\left|X_{i}\right|^{p}+\left(\sum_{i=1}^{n} E X_{i}^{2}\right)^{p / 2}\right],
$$

for every $n \geq 1$.

\section{Main Results and Their Proofs}

Let $\left\{X_{n k}, k \geq 1, n \geq 1\right\}$ be an array of rowwise $\varphi$-mixing random variables and let $\varphi_{n}(\cdot)$ be the mixing coefficient of $\left\{X_{n k}, k \geq 1\right\}$ for any $n \geq 1$. Our main results are as follows.

Theorem 4. Let $\left\{X_{n k}, k \geq 1, n \geq 1\right\}$ be an array of rowwise $\varphi$ mixing random variables satisfying $\sup _{n \geq 1} \sum_{k=1}^{\infty} \varphi_{n}^{1 / 2}(k)<\infty$ and let $\left\{a_{n}, n \geq 1\right\}$ be a sequence of positive real numbers such that $a_{n} \uparrow \infty$. Also, let $\left\{\Psi_{k}(t), k \geq 1\right\}$ be a positive even function satisfying (5) for $1 \leq q<p \leq 2$. Then under conditions (6) and (7), one has

$$
\sum_{n=1}^{\infty} a_{n}^{-q} E\left\{\max _{1 \leq j \leq n}\left|\sum_{k=1}^{j} X_{n k}\right|-\varepsilon a_{n}\right\}_{+}^{q}<\infty, \quad \forall \varepsilon>0 .
$$

Proof. Firstly, let us prove the following statements from conditions (5) and (7).

(i) For $r \geq 1,0<u \leq q$,

$$
\begin{aligned}
& \sum_{n=1}^{\infty}\left(\sum_{k=1}^{n} \frac{E\left|X_{n k}\right|^{u} I\left(\left|X_{n k}\right|>a_{n}\right)}{a_{n}^{u}}\right)^{r} \\
& \quad \leq \sum_{n=1}^{\infty}\left(\sum_{k=1}^{n} \frac{E\left|X_{n k}\right|^{q} I\left(\left|X_{n k}\right|>a_{n}\right)}{a_{n}^{q}}\right)^{r} \\
& \quad \leq \sum_{n=1}^{\infty}\left(\sum_{k=1}^{n} E \frac{\Psi_{k}\left(X_{n k}\right)}{\Psi_{k}\left(a_{n}\right)}\right)^{r} \\
& \quad \leq\left(\sum_{n=1}^{\infty} \sum_{k=1}^{n} E \frac{\Psi_{k}\left(X_{n k}\right)}{\Psi_{k}\left(a_{n}\right)}\right)^{r}<\infty .
\end{aligned}
$$

(ii) For $v \geq p$,

$$
\begin{aligned}
\sum_{n=1}^{\infty} & \sum_{k=1}^{n} \frac{E\left|X_{n k}\right|^{v} I\left(\left|X_{n k}\right| \leq a_{n}\right)}{a_{n}^{v}} \\
& \leq \sum_{n=1}^{\infty} \sum_{k=1}^{n} \frac{E\left|X_{n k}\right|^{p} I\left(\left|X_{n k}\right| \leq a_{n}\right)}{a_{n}^{p}} \\
& \leq \sum_{n=1}^{\infty} \sum_{k=1}^{n} E \frac{\Psi_{k}\left(X_{n k}\right)}{\Psi_{k}\left(a_{n}\right)}<\infty .
\end{aligned}
$$


For $n \geq 1$, denote $M_{n}(X)=\max _{1 \leq j \leq n}\left|\sum_{k=1}^{j} X_{n k}\right|$. It is easy to check that

$$
\begin{aligned}
\sum_{n=1}^{\infty} a_{n}^{-q} E\left\{M_{n}(X)-\varepsilon a_{n}\right\}_{+}^{q} \\
=\sum_{n=1}^{\infty} a_{n}^{-q} \int_{0}^{\infty} P\left\{M_{n}(X)-\varepsilon a_{n}>t^{1 / q}\right\} d t \\
=\sum_{n=1}^{\infty} a_{n}^{-q}\left(\int_{0}^{a_{n}^{q}} P\left\{M_{n}(X)>\varepsilon a_{n}+t^{1 / q}\right\} d t\right. \\
\quad+\sum_{n=1}^{\infty} P\left\{M_{n}(X)>\varepsilon a_{n}\right\} \\
\quad+\sum_{n=1}^{\infty} a_{n}^{-q} \int_{a_{n}^{q}}^{\infty} P\left\{M_{n}(X)>t^{1 / q}\right\} d t \doteq I_{1}+I_{2} .
\end{aligned}
$$

To prove (14), it suffices to prove that $I_{1}<\infty$ and $I_{2}<\infty$. Now let us prove them step by step. Firstly, we prove that $I_{1}<$ $\infty$.

For all $n \geq 1$, define

$$
X_{k}^{(n)}=X_{n k} I\left(\left|X_{n k}\right| \leq a_{n}\right), \quad T_{j}^{(n)}=\frac{1}{a_{n}} \sum_{k=1}^{j}\left(X_{k}^{(n)}-E X_{k}^{(n)}\right),
$$

then for all $\varepsilon>0$, it is easy to have

$$
\begin{aligned}
P\left(\max _{1 \leq j \leq n}\left|\frac{1}{a_{n}} \sum_{k=1}^{j} X_{n k}\right|>\varepsilon\right) \\
\leq P\left(\max _{1 \leq j \leq n}\left|X_{n k}\right|>a_{n}\right) \\
\quad+P\left(\max _{1 \leq j \leq n}\left|T_{j}^{(n)}\right|>\varepsilon-\max _{1 \leq j \leq n}\left|\frac{1}{a_{n}} \sum_{k=1}^{j} E X_{k}^{(n)}\right|\right) .
\end{aligned}
$$

By (5), (6), (7), and (15) we have

$$
\begin{aligned}
& \max _{1 \leq j \leq n}\left|\frac{1}{a_{n}} \sum_{k=1}^{j} E X_{k}^{(n)}\right| \\
& =\max _{1 \leq j \leq n}\left|\frac{1}{a_{n}} \sum_{k=1}^{j} E X_{n k} I\left(\left|X_{n k}\right| \leq a_{n}\right)\right| \\
& =\max _{1 \leq j \leq n}\left|\frac{1}{a_{n}} \sum_{k=1}^{j} E X_{n k} I\left(\left|X_{n k}\right|>a_{n}\right)\right| \\
& \leq \sum_{k=1}^{n} \frac{E\left|X_{n k}\right| I\left(\left|X_{n k}\right|>a_{n}\right)}{a_{n}} \longrightarrow 0 \text { as } n \longrightarrow \infty \text {. }
\end{aligned}
$$

From (19) and (20), it follows that, for $n$ large enough,

$$
\begin{aligned}
& P\left(\max _{1 \leq j \leq n}\left|\frac{1}{a_{n}} \sum_{k=1}^{j} X_{n k}\right|>\varepsilon\right) \\
& \quad \leq \sum_{k=1}^{n} P\left(\left|X_{n k}\right|>a_{n}\right)+P\left(\max _{1 \leq j \leq n}\left|T_{j}^{(n)}\right|>\frac{\varepsilon}{2}\right) .
\end{aligned}
$$

Hence we only need to prove that

$$
\begin{gathered}
I \doteq \sum_{n=1}^{\infty} \sum_{k=1}^{n} P\left(\left|X_{n k}\right|>a_{n}\right)<\infty, \\
I I \doteq \sum_{n=1}^{\infty} P\left(\max _{1 \leq j \leq n}\left|T_{j}^{(n)}\right|>\frac{\varepsilon}{2}\right)<\infty .
\end{gathered}
$$

For $I$, it follows by (15) that

$$
\begin{aligned}
I & =\sum_{n=1}^{\infty} \sum_{k=1}^{n} E I\left(\left|X_{n k}\right|>a_{n}\right) \\
& \leq \sum_{n=1}^{\infty} \sum_{k=1}^{n} \frac{E\left|X_{n k}\right|^{q} I\left(\left|X_{n k}\right|>a_{n}\right)}{a_{n}^{q}}<\infty .
\end{aligned}
$$

For $I I$, take $r \geq 2$. Since $p \leq 2, r \geq p$, we have by Markov inequality, Lemma $3, C_{r}$-inequality, and (16) that

$$
\begin{aligned}
I I & \leq \sum_{n=1}^{\infty}\left(\frac{\varepsilon}{2}\right)^{-r} E \max _{1 \leq j \leq n}\left|T_{j}^{(n)}\right|^{r} \\
\leq & C \sum_{n=1}^{\infty}\left(\frac{\varepsilon}{2}\right)^{-r} \frac{1}{a_{n}^{r}}\left[\sum_{k=1}^{n} E\left|X_{k}^{(n)}\right|^{r}+\left(\sum_{k=1}^{n} E\left|X_{k}^{(n)}\right|^{2}\right)^{r / 2}\right] \\
\leq & C \sum_{n=1}^{\infty} \sum_{k=1}^{n} \frac{E\left|X_{k}^{(n)}\right|^{r}}{a_{n}^{r}}+C \sum_{n=1}^{\infty}\left(\sum_{k=1}^{n} \frac{E\left|X_{k}^{(n)}\right|^{2}}{a_{n}^{2}}\right)^{r / 2} \\
\leq & C \sum_{n=1}^{\infty} \sum_{k=1}^{n} \frac{E\left|X_{n k}\right|^{p} I\left(\left|X_{n k}\right| \leq a_{n}\right)}{a_{n}^{p}} \\
& +C \sum_{n=1}^{\infty}\left(\sum_{k=1}^{n} \frac{E\left|X_{n k}\right|^{p} I\left(\left|X_{n k}\right| \leq a_{n}\right)}{a_{n}^{p}}\right)^{r / 2} \\
\leq & C \sum_{n=1}^{\infty} \sum_{k=1}^{n} \frac{E\left|X_{n k}\right|^{p} I\left(\left|X_{n k}\right| \leq a_{n}\right)}{a_{n}^{p}} \\
& +C\left(\sum_{n=1}^{\infty} \sum_{k=1}^{n} \frac{E\left|X_{n k}\right|^{p} I\left(\left|X_{n k}\right| \leq a_{n}\right)}{a_{n}^{p}}\right)^{r / 2}<.
\end{aligned}
$$

Next we prove that $I_{2}<\infty$. Denote $Y_{n k}=X_{n k} I\left(\left|X_{n k}\right| \leq t^{1 / q}\right)$, $Z_{n k}=X_{n k}-Y_{n k}$, and $M_{n}(Y)=\max _{1 \leq j \leq n}\left|\sum_{k=1}^{j} Y_{n k}\right|$. Obviously,

$$
\begin{aligned}
& P\left\{M_{n}(X)>t^{1 / q}\right\} \\
& \quad \leq \sum_{k=1}^{n} P\left\{\left|X_{n k}\right|>t^{1 / q}\right\}+P\left\{M_{n}(Y)>t^{1 / q}\right\} .
\end{aligned}
$$


Hence,

$$
\begin{aligned}
I_{2} \leq & \sum_{n=1}^{\infty} \sum_{k=1}^{n} a_{n}^{-q} \int_{a_{n}^{q}}^{\infty} P\left\{\left|X_{n k}\right|>t^{1 / q}\right\} d t \\
& +\sum_{n=1}^{\infty} a_{n}^{-q} \int_{a_{n}^{q}}^{\infty} P\left\{M_{n}(Y)>t^{1 / q}\right\} d t \\
\doteq & I_{3}+I_{4} .
\end{aligned}
$$

For $I_{3}$, by (15), we have

$$
\begin{aligned}
I_{3} & =\sum_{n=1}^{\infty} \sum_{k=1}^{n} a_{n}^{-q} \int_{a_{n}^{q}}^{\infty} P\left\{\left|X_{n k}\right| I\left(\left|X_{n k}\right|>a_{n}\right)>t^{1 / q}\right\} d t \\
& \leq \sum_{n=1}^{\infty} \sum_{k=1}^{n} a_{n}^{-q} \int_{0}^{\infty} P\left\{\left|X_{n k}\right| I\left(\left|X_{n k}\right|>a_{n}\right)>t^{1 / q}\right\} d t \\
& =\sum_{n=1}^{\infty} \sum_{k=1}^{n} \frac{E\left|X_{n k}\right|^{q} I\left(\left|X_{n k}\right|>a_{n}\right)}{a_{n}^{q}}<\infty .
\end{aligned}
$$

Now let us prove that $I_{4}<\infty$. Firstly, it follows by (6) and (15) that

$$
\begin{aligned}
& \max _{t \geq a_{n}^{q}} \max _{1 \leq j \leq n} t^{-1 / q}\left|\sum_{k=1}^{j} E Y_{n k}\right| \\
& \quad=\max _{t \geq a_{n}^{q}} \max _{1 \leq j \leq n} t^{-1 / q}\left|\sum_{k=1}^{j} E Z_{n k}\right| \\
& \leq \max _{t \geq a_{n}^{q}} t^{-1 / q} \sum_{k=1}^{n} E\left|X_{n k}\right| I\left(\left|X_{n k}\right|>t^{1 / q}\right) \\
& \leq \sum_{k=1}^{n} a_{n}^{-1} E\left|X_{n k}\right| I\left(\left|X_{n k}\right|>a_{n}\right) \\
& \leq \sum_{k=1}^{n} \frac{E\left|X_{n k}\right|^{q} I\left(\left|X_{n k}\right|>a_{n}\right)}{a_{n}^{q}} \longrightarrow 0 \text { as } n \longrightarrow \infty .
\end{aligned}
$$

Therefore, for $n$ sufficiently large,

$$
\max _{1 \leq j \leq n}\left|\sum_{k=1}^{j} E Y_{n k}\right| \leq \frac{t^{1 / q}}{2}, \quad t \geq a_{n}^{q} .
$$

Then for $n$ sufficiently large,

$$
\begin{aligned}
& P\left\{M_{n}(Y)>t^{1 / q}\right\} \\
& \quad \leq P\left\{\max _{1 \leq j \leq n}\left|\sum_{k=1}^{j}\left(Y_{n k}-E Y_{n k}\right)\right|>\frac{t^{1 / q}}{2}\right\}, \quad t \geq a_{n}^{q} .
\end{aligned}
$$

Let $d_{n}=\left[a_{n}\right]+1$. By (30), Lemma 3, and $C_{r}$-inequality, we can see that

$$
\begin{aligned}
I_{4} \leq & C \sum_{n=1}^{\infty} a_{n}^{-q} \int_{a_{n}^{q}}^{\infty} t^{-2 / q} E\left(\max _{1 \leq j \leq n}\left|\sum_{k=1}^{j}\left(Y_{n k}-E Y_{n k}\right)\right|\right)^{2} d t \\
\leq & C \sum_{n=1}^{\infty} a_{n}^{-q} \int_{a_{n}^{q}}^{\infty} t^{-2 / q} \sum_{k=1}^{n} E\left(Y_{n k}-E Y_{n k}\right)^{2} d t \\
\leq & C \sum_{n=1}^{\infty} \sum_{k=1}^{n} a_{n}^{-q} \int_{a_{n}^{q}}^{\infty} t^{-2 / q} E Y_{n k}^{2} d t \\
= & C \sum_{n=1}^{\infty} \sum_{k=1}^{n} a_{n}^{-q} \int_{a_{n}^{q}}^{\infty} t^{-2 / q} E X_{n k}^{2} I\left(\left|X_{n k}\right| \leq d_{n}\right) d t \\
& +C \sum_{n=1}^{\infty} \sum_{k=1}^{n} a_{n}^{-q} \int_{d_{n}^{q}}^{\infty} t^{-2 / q} E X_{n k}^{2} I\left(d_{n}<\left|X_{n k}\right| \leq t^{1 / q}\right) d t \\
\doteq & I_{41}+I_{42} .
\end{aligned}
$$

For $I_{41}$, since $q<2$, we have

$$
\begin{aligned}
I_{41}= & C \sum_{n=1}^{\infty} \sum_{k=1}^{n} a_{n}^{-q} E X_{n k}^{2} I\left(\left|X_{n k}\right| \leq d_{n}\right) \int_{a_{n}^{q}}^{\infty} t^{-2 / q} d t \\
\leq & C \sum_{n=1}^{\infty} \sum_{k=1}^{n} \frac{E X_{n k}^{2} I\left(\left|X_{n k}\right| \leq d_{n}\right)}{a_{n}^{2}} \\
= & C \sum_{n=1}^{\infty} \sum_{k=1}^{n} \frac{E X_{n k}^{2} I\left(\left|X_{n k}\right| \leq a_{n}\right)}{a_{n}^{2}} \\
& +C \sum_{n=1}^{\infty} \sum_{k=1}^{n} \frac{E X_{n k}^{2} I\left(a_{n}<\left|X_{n k}\right| \leq d_{n}\right)}{a_{n}^{2}} \\
\doteq & I_{41}^{\prime}+I_{41}^{\prime \prime} .
\end{aligned}
$$

Since $p \leq 2$, by (16), it implies $I_{41}^{\prime}<\infty$. Now we prove that $I_{41}^{\prime \prime}<\infty$. Since $q<2$ and $\left(a_{n}+1\right) / a_{n} \rightarrow 1$ as $n \rightarrow \infty$, by (15) we have

$$
\begin{aligned}
I_{41}^{\prime \prime} & \leq C \sum_{n=1}^{\infty} \sum_{k=1}^{n} \frac{d_{n}^{2-q}}{a_{n}^{2}} E\left|X_{n k}\right|^{q} I\left(a_{n}<\left|X_{n k}\right| \leq d_{n}\right) \\
& \leq C \sum_{n=1}^{\infty} \sum_{k=1}^{n}\left(\frac{a_{n}+1}{a_{n}}\right)^{2-q} \frac{E\left|X_{n k}\right|^{q} I\left(\left|X_{n k}\right|>a_{n}\right)}{a_{n}^{q}} \\
& \leq C \sum_{n=1}^{\infty} \sum_{k=1}^{n} \frac{E\left|X_{n k}\right|^{q} I\left(\left|X_{n k}\right|>a_{n}\right)}{a_{n}^{q}}<\infty .
\end{aligned}
$$


Let $t=u^{q}$ in $I_{42}$. Note that, for $q<2$,

$$
\begin{aligned}
& \int_{d_{n}}^{\infty} u^{q-3} E X_{n k}^{2} I\left(d_{n}<\left|X_{n k}\right| \leq u\right) d u \\
& \quad=\int_{d_{n}}^{\infty} u^{q-3} E X_{n k}^{2} I\left(\left|X_{n k}\right|>d_{n}\right) \cdot I\left(\left|X_{n k}\right| \leq u\right) d u \\
& \quad=E\left[X_{n k}^{2} I\left(\left|X_{n k}\right|>d_{n}\right) \int_{\left|X_{n k}\right|}^{\infty} u^{q-3} I\left(\left|X_{n k}\right| \leq u\right) d u\right] \\
& \quad=E\left[X_{n k}^{2} I\left(\left|X_{n k}\right|>d_{n}\right) \int_{\left|X_{n k}\right|}^{\infty} u^{q-3} d u\right] \\
& \quad \leq C E\left|X_{n k}\right|^{q} I\left(\left|X_{n k}\right|>d_{n}\right) .
\end{aligned}
$$

Then by (15) and $d_{n}>a_{n}$, we have

$$
\begin{aligned}
I_{42} & =C \sum_{n=1}^{\infty} \sum_{k=1}^{n} a_{n}^{-q} \int_{d_{n}}^{\infty} u^{q-3} E X_{n k}^{2} I\left(d_{n}<\left|X_{n k}\right| \leq u\right) d u \\
& \leq C \sum_{n=1}^{\infty} \sum_{k=1}^{n} a_{n}^{-q} E\left|X_{n k}\right|^{q} I\left(\left|X_{n k}\right|>a_{n}\right)<\infty
\end{aligned}
$$

This completes the proof of Theorem 4 .

Theorem 5. Let $\left\{X_{n k}, k \geq 1, n \geq 1\right\}$ be an array of rowwise $\varphi$ mixing random variables satisfying $\sup _{n \geq 1} \sum_{k=1}^{\infty} \varphi_{n}^{1 / 2}(k)<\infty$ and let $\left\{a_{n}, n \geq 1\right\}$ be a sequence of positive real numbers such that $a_{n} \uparrow \infty$. Also, let $\left\{\Psi_{k}(t), k \geq 1\right\}$ be a positive even function satisfying (5) for $1 \leq q<p$ and $p>2$. Then conditions (6)-(8) imply (14).

Proof. Following the notation, by a similar argument as in the proof of Theorem 4 , we can easily prove that $I_{1}<\infty, I_{3}<\infty$ and that (19) and (20) hold. To complete the proof, we only need to prove that $I_{4}<\infty$.

Let $\delta \geq p$ and $d_{n}=\left[a_{n}\right]+1$. By (30), Markov inequality, Lemma 3 , and the $C_{r}$-inequality we can get

$$
\begin{aligned}
I_{4} \leq & C \sum_{n=1}^{\infty} a_{n}^{-q} \int_{a_{n}^{q}}^{\infty} t^{-\delta / q} \operatorname{Emax}_{1 \leq j \leq n}\left|\sum_{k=1}^{j}\left(Y_{n k}-E Y_{n k}\right)\right|^{\delta} d t \\
\leq & C \sum_{n=1}^{\infty} a_{n}^{-q} \int_{a_{n}^{q}}^{\infty} t^{-\delta / q}\left[\sum_{k=1}^{n} E\left|Y_{n k}\right|^{\delta}+\left(\sum_{k=1}^{n} E Y_{n k}^{2}\right)^{\delta / 2}\right] d t \\
= & C \sum_{n=1}^{\infty} \sum_{k=1}^{n} a_{n}^{-q} \int_{a_{n}^{q}}^{\infty} t^{-\delta / q} E\left|Y_{n k}\right|^{\delta} d t \\
& +C \sum_{n=1}^{\infty} a_{n}^{-q} \int_{a_{n}^{q}}^{\infty} t^{-\delta / q}\left(\sum_{k=1}^{n} E Y_{n k}^{2}\right)^{\delta / 2} d t \\
\doteq & I_{43}+I_{44} .
\end{aligned}
$$

For $I_{43}$, we have

$$
\begin{aligned}
I_{43}= & C \sum_{n=1}^{\infty} \sum_{k=1}^{n} a_{n}^{-q} \int_{a_{n}^{q}}^{\infty} t^{-\delta / q} E\left|X_{n k}\right|^{\delta} I\left(\left|X_{n k}\right| \leq d_{n}\right) d t \\
& +C \sum_{n=1}^{\infty} \sum_{k=1}^{n} a_{n}^{-q} \int_{d_{n}^{q}}^{\infty} t^{-\delta / q} E\left|X_{n k}\right|^{\delta} I\left(d_{n}<\left|X_{n k}\right| \leq t^{1 / q}\right) d t \\
\doteq & I_{43}^{\prime}+I_{43}^{\prime \prime} .
\end{aligned}
$$

By a similar argument as in the proof of $I_{41}<\infty$ and $I_{42}<$ $\infty$ (replacing the exponent 2 by $\delta$ ), we can get $I_{43}^{\prime}<\infty$ and $I_{43}^{\prime \prime}<\infty$.

For $I_{44}$, since $\delta>2$, we can see that

$$
\begin{aligned}
I_{44}= & C \sum_{n=1}^{\infty} a_{n}^{-q} \int_{a_{n}^{q}}^{\infty} t^{-\delta / q}\left(\sum_{k=1}^{n} E X_{n k}^{2} I\left(\left|X_{n k}\right| \leq a_{n}\right)\right. \\
& \left.\quad+\sum_{k=1}^{n} E X_{n k}^{2} I\left(a_{n}<\left|X_{n k}\right| \leq t^{1 / q}\right)\right)^{\delta / 2} d t \\
\leq & C \sum_{n=1}^{\infty} a_{n}^{-q} \int_{a_{n}^{q}}^{\infty} t^{-\delta / q}\left(\sum_{k=1}^{n} E X_{n k}^{2} I\left(\left|X_{n k}\right| \leq a_{n}\right)\right)^{\delta / 2} d t \\
& +C \sum_{n=1}^{\infty} a_{n}^{-q} \int_{a_{n}^{q}}^{\infty}\left(t^{-2 / q} \sum_{k=1}^{n} E X_{n k}^{2} I\left(a_{n}<\left|X_{n k}\right| \leq t^{1 / q}\right)\right)^{\delta / 2} d t \\
\doteq & I_{44}^{\prime}+I_{44}^{\prime \prime} .
\end{aligned}
$$

Since $\delta \geq p>q$, from (8) we have

$$
\begin{aligned}
I_{44}^{\prime} & =C \sum_{n=1}^{\infty} a_{n}^{-q}\left(\sum_{k=1}^{n} E X_{n k}^{2} I\left(\left|X_{n k}\right| \leq a_{n}\right)\right)^{\delta / 2} \int_{a_{n}^{q}}^{\infty} t^{-\delta / q} d t \\
& \leq C \sum_{n=1}^{\infty}\left(\sum_{k=1}^{n} \frac{E X_{n k}^{2} I\left(\left|X_{n k}\right| \leq a_{n}\right)}{a_{n}^{2}}\right)^{\delta / 2} \\
& \leq C \sum_{n=1}^{\infty}\left(\sum_{k=1}^{n} \frac{E X_{n k}^{2}}{a_{n}^{2}}\right)^{\delta / 2}<\infty .
\end{aligned}
$$

Next we prove that $I_{44}^{\prime \prime}<\infty$. To start with, we consider the case $1 \leq q \leq 2$. Since $\delta>2$, by (15), we have

$$
\begin{aligned}
I_{44}^{\prime \prime} & \leq C \sum_{n=1}^{\infty} a_{n}^{-q} \int_{a_{n}^{q}}^{\infty}\left(t^{-1} \sum_{k=1}^{n} E\left|X_{n k}\right|^{q} I\left(a_{n}<\left|X_{n k}\right| \leq t^{1 / q}\right)\right)^{\delta / 2} d t \\
& \leq C \sum_{n=1}^{\infty} a_{n}^{-q} \int_{a_{n}^{q}}^{\infty}\left(t^{-1} \sum_{k=1}^{n} E\left|X_{n k}\right|^{q} I\left(\left|X_{n k}\right|>a_{n}\right)\right)^{\delta / 2} d t
\end{aligned}
$$




$$
\begin{aligned}
& =C \sum_{n=1}^{\infty} a_{n}^{-q}\left(\sum_{k=1}^{n} E\left|X_{n k}\right|^{q} I\left(\left|X_{n k}\right|>a_{n}\right)\right)^{\delta / 2} \int_{a_{n}^{q}}^{\infty} t^{-\delta / 2} d t \\
& \leq C \sum_{n=1}^{\infty}\left(\sum_{k=1}^{n} \frac{E\left|X_{n k}\right|^{q} I\left(\left|X_{n k}\right|>a_{n}\right)}{a_{n}^{q}}\right)^{\delta / 2}<\infty .
\end{aligned}
$$

Finally, we prove that $I_{44}^{\prime \prime}<\infty$ in the case $2<q<p$. Since $\delta>q$ and $\delta>2$, we have by (15) that

$$
\begin{aligned}
I_{44}^{\prime \prime} & \leq C \sum_{n=1}^{\infty} a_{n}^{-q} \int_{a_{n}^{q}}^{\infty}\left(t^{-2 / q} \sum_{k=1}^{n} E X_{n k}^{2} I\left(\left|X_{n k}\right|>a_{n}\right)\right)^{\delta / 2} d t \\
& =C \sum_{n=1}^{\infty} a_{n}^{-q}\left(\sum_{k=1}^{n} E X_{n k}^{2} I\left(\left|X_{n k}\right|>a_{n}\right)\right)^{\delta / 2} \int_{a_{n}^{q}}^{\infty} t^{-\delta / q} d t \\
& \leq C \sum_{n=1}^{\infty}\left(\sum_{k=1}^{n} \frac{E X_{n k}^{2} I\left(\left|X_{n k}\right|>a_{n}\right)}{a_{n}^{2}}\right)^{\delta / 2}<\infty .
\end{aligned}
$$

Thus we get the desired result immediately. The proof is completed.

Corollary 6. Let $\left\{X_{n k}, k \geq 1, n \geq 1\right\}$ be an array of rowwise $\varphi$-mixing mean zero random variables with $\sup _{n \geq 1} \sum_{k=1}^{\infty} \varphi_{n}^{1 / 2}(k)<\infty, q \geq 1$. If, for some $\alpha>0$ and $v \geq 2$,

$$
\max _{1 \leq k \leq n} E\left|X_{n k}\right|^{\nu}=O\left(n^{\alpha}\right),
$$

where $(v / q)-\alpha>\max \{v / 2,2\}, v \geq 2$, then, for any $\varepsilon>0$,

$$
\sum_{n=1}^{\infty} n^{-1} E\left\{\max _{1 \leq j \leq n}\left|\sum_{k=1}^{j} X_{n k}\right|-\varepsilon n^{1 / q}\right\}_{+}^{q}<\infty .
$$

Proof. Put $\Psi_{k}(|t|)=|t|^{v}, p=v+\delta, \delta>0$, and $a_{n}=n^{1 / q}$.

Since $v \geq 2,(v / q)-\alpha>\max \{v / r, 2\}$, then

$\frac{\Psi_{k}(|t|)}{|t|^{q}}=|t|^{\nu-q} \uparrow, \quad \frac{\Psi_{k}(|t|)}{|t|^{p}}=\frac{|t|^{\nu}}{|t|^{p}}=\frac{1}{|t|^{\delta}} \downarrow \quad$ as $|t| \uparrow \infty$.

It follows by (42) and $(v / q)-\alpha>2$ that

$$
\sum_{n=1}^{\infty} \sum_{k=1}^{n} \frac{E \Psi_{k}\left(X_{n k}\right)}{\Psi_{k}\left(a_{n}\right)}=\sum_{n=1}^{\infty} \sum_{k=1}^{n} \frac{E\left|X_{n k}\right|^{v}}{n^{v / q}} \leq C \sum_{n=1}^{\infty} \frac{1}{n^{(v / q)-\alpha-1}}<\infty .
$$

Since $v \geq 2$, by Jensen's inequality it follows that

$$
\sum_{k=1}^{n} \frac{E\left|X_{n k}\right|^{2}}{n^{2 / q}} \leq \sum_{k=1}^{n} \frac{\left(E\left|X_{n k}\right|^{v}\right)^{2 / v}}{n^{2 / q}} \leq C \frac{1}{n^{(2 / q)-(2 \alpha / v)-1}} .
$$

Clearly $(2 / q)-(2 \alpha / v)-1>0$. Take $s>p$ such that $(s / 2)((2 / q)-(2 \alpha / v)-1)>1$. Therefore,

$$
\sum_{n=1}^{\infty}\left[\sum_{k=1}^{n} \frac{E\left|X_{n k}\right|^{2}}{n^{2 / q}}\right]^{s / 2}<\infty
$$

Combining Theorem 5 and (45)-(47), we can prove Corollary 6 immediately.

Remark 7. Noting that in this paper we consider the case $1 \leq$ $q \leq p$, which has a more wide scope than the case $q=1$, $p \geq 2$ in Gan et al. [14]. In addition, compared with $\varphi$-mixing random variables, the arrays of $\varphi$-mixing random variables not only have many related properties, but also have a wide range of application. So it is very significant to study it.

Remark 8. Under the condition of Theorem 4, we have

$$
\begin{aligned}
\infty & >\sum_{n=1}^{\infty} a_{n}^{-q} E\left\{\max _{1 \leq j \leq n}\left|\sum_{k=1}^{j} X_{n k}\right|-\varepsilon a_{n}\right\}_{+}^{q} \\
& =\sum_{n=1}^{\infty} a_{n}^{-q} \int_{0}^{\infty} P\left\{\max _{1 \leq j \leq n}\left|\sum_{k=1}^{j} X_{n k}\right|-\varepsilon a_{n}>t^{1 / q}\right\} d t \\
& \geq \sum_{n=1}^{\infty} a_{n}^{-q} \int_{0}^{\varepsilon^{q} a_{n}^{q}} P\left\{\max _{1 \leq j \leq n}\left|\sum_{k=1}^{j} X_{n k}\right|-\varepsilon a_{n}>\varepsilon a_{n}\right\} d t \\
& =\varepsilon^{q} \sum_{n=1}^{\infty} P\left\{\max _{1 \leq j \leq n}\left|\sum_{k=1}^{j} X_{n k}\right|>2 \varepsilon a_{n}\right\} .
\end{aligned}
$$

Then we can obtain (11) directly. In this case, condition (10) is not needed. Especially, for $p=2$, the conditions of Theorem 4 are weaker than Theorem A. So Theorem 4 generalizes and improves it.

Remark 9. Note that Theorem A only considers $q=1$, while Theorem 5 considers $q \geq 1$. In addition, (14) implies (11), so Theorem 5 generalizes the corresponding result of Theorem A.

\section{Conflict of Interests}

The authors declare that there is no conflict of interests regarding the publication of this paper.

\section{Acknowledgments}

The authors are most grateful to the Editor Ming Mei and anonymous reviewer for careful reading of the paper and valuable suggestions which helped in improving an earlier version of this paper. This work was supported by National Natural Science Foundation of China $(11201001,11126176)$ and the Students Innovative Training Project of Anhui University (201310357004).

\section{References}

[1] R. L. Dobrushin, "The central limit theorem for non-stationary Markov chain," Theory of Probability and Its Applications, vol. 1, pp. 72-88, 1956.

[2] S. A. Utev, "The central limit theorem for $\varphi$-mixing arrays of random variables," Theory of Probability and Its Applications, vol. 35, no. 1, pp. 131-139, 1990. 
[3] S. X. Gan and P. Y. Chen, "Some limit theorems for sequences of pairwise NQD random variables," Acta Mathematica Scientia, vol. 28, no. 2, pp. 269-281, 2008.

[4] M. Peligrad, "An invariance principle for $\varphi$-mixing sequences," The Annals of Probability, vol. 13, no. 4, pp. 1304-1313, 1985.

[5] Q.-M. Shao, "Almost sure invariance principles for mixing sequences of random variables," Stochastic Processes and Their Applications, vol. 48, no. 2, pp. 319-334, 1993.

[6] P. Y. Chen and D. C. Wang, "Complete moment convergence for sequence of identically distributed $\varphi$-mixing random variables," Acta Mathematica Sinica, vol. 26, no. 4, pp. 679-690, 2010.

[7] A. T. Shen, X. H. Wang, and J. M. Ling, "On complete convergence for non-stationary $\varphi$-mixingrandom variables," Communications in Statistics-Theory and Methods. In press.

[8] A. T. Shen, X. H. Wang, and X. Q. Li, "On the rate of completeconvergence for weighted sums of arrays of rowwise $\varphi$ mixing random variables," Communications in Statistics-Theory and Methods. In press.

[9] Q. Y. Wu, Probability Limit Theory for Mixing Sequences, Science Press of China, Beijing, China, 2006.

[10] X. J. Wang, S. H. Hu, W. Z. Yang, and Y. Shen, "On complete convergence for weighted sums of $\varphi$-mixing random variables," Journal of Inequalities and Applications, vol. 2010, Article ID 372390, 2010.

[11] S. H. Hu and X. J. Wang, "Large deviations for some dependent sequences," Acta Mathematica Scientia, vol. 28, no. 2, pp. 295300, 2008.

[12] P. L. Hsu and H. Robbins, "Complete convergence and the law of large numbers," Proceedings of the National Academy of Sciences of the United States of America, vol. 33, no. 2, pp. 25-31, 1947.

[13] Y.S. Chow, "On the rate of moment convergence of sample sums and extremes," Bulletin of the Institute of Mathematics Academia Sinica, vol. 16, no. 3, pp. 177-201, 1988.

[14] S. Gan, P. Chen, and D. Qiu, "Strong law of large numbers and complete convergence for sequences of $\varphi$-mixing random variables," Wuhan University Journal of Natural Sciences, vol. 12, no. 2, pp. 211-217, 2007.

[15] Y. Wu, C. Wang, and A. Volodin, "Limiting behavior for arrays of rowwise $\rho^{*}$-mixing random variables," Lithuanian Mathematical Journal, vol. 52, no. 2, pp. 214-221, 2012.

[16] Y. F. Wu, "On limiting behavior for arrays of rowwise negatively orthant dependent random variables," Journal of the Korean Statistical Society, vol. 42, pp. 61-70, 2013.

[17] H. Huang, D. Wang, and Q. Wu, "Strong convergence laws for $\widetilde{\varphi}$-mixing sequences of random variables," Chinese Journal of Applied Probability and Statistics, vol. 28, no. 2, pp. 181-188, 2012.

[18] A. T. Shen, "Some strong limit theorems for arrays of rowwise negatively orthant-dependent random variables," Journal of Inequalities and Applications, vol. 2011, Article ID 93, 2011.

[19] A. T. Shen, R. C. Wu, Y. Chen, and Y. Zhou, "Complete convergence of the maximum partial sums for arrays of rowwise of AANA random variables," Discrete Dynamics in Nature and Society, vol. 2013, Article ID 741901, 7 pages, 2013.

[20] A. T. Shen, R. C. Wu, X. H. Wang, and Y. Shen, "Complete convergence for weighted sums of arrays of rowwise $\tilde{\rho}$-mixing random variables," Journal of Inequalities and Applications, vol. 2013, Article ID 356, 2013. 


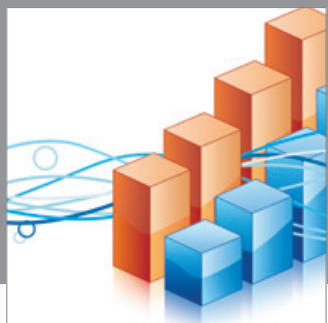

Advances in

Operations Research

mansans

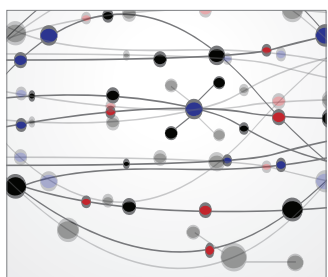

The Scientific World Journal
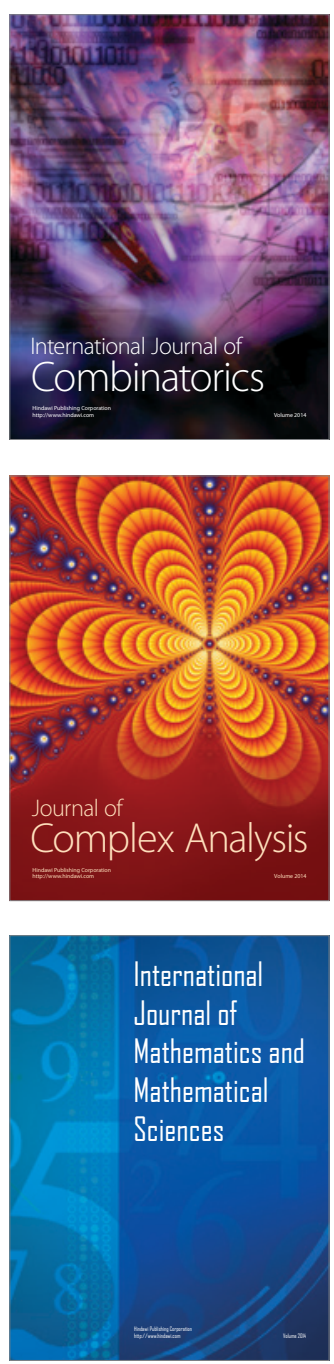
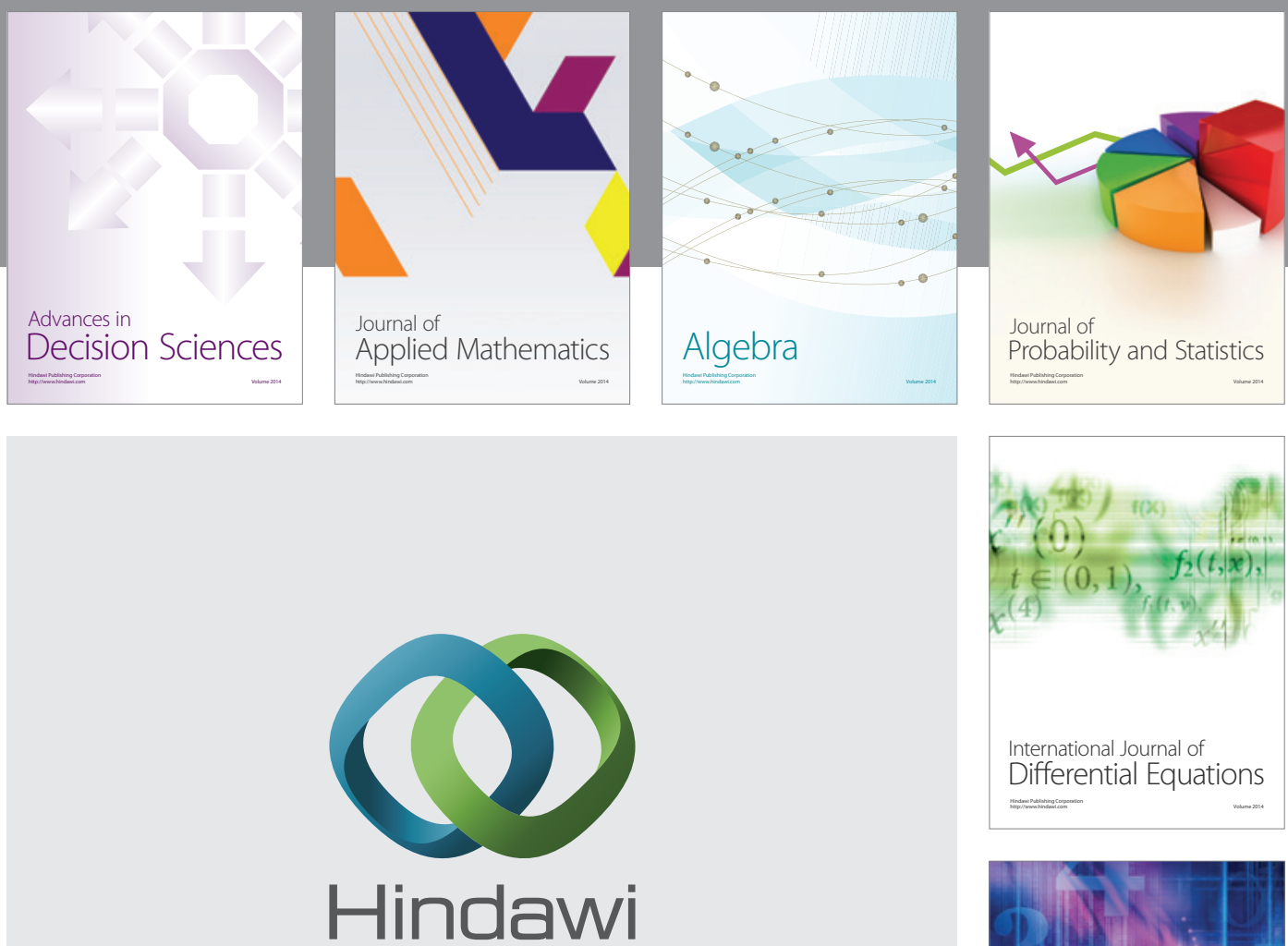

Submit your manuscripts at http://www.hindawi.com
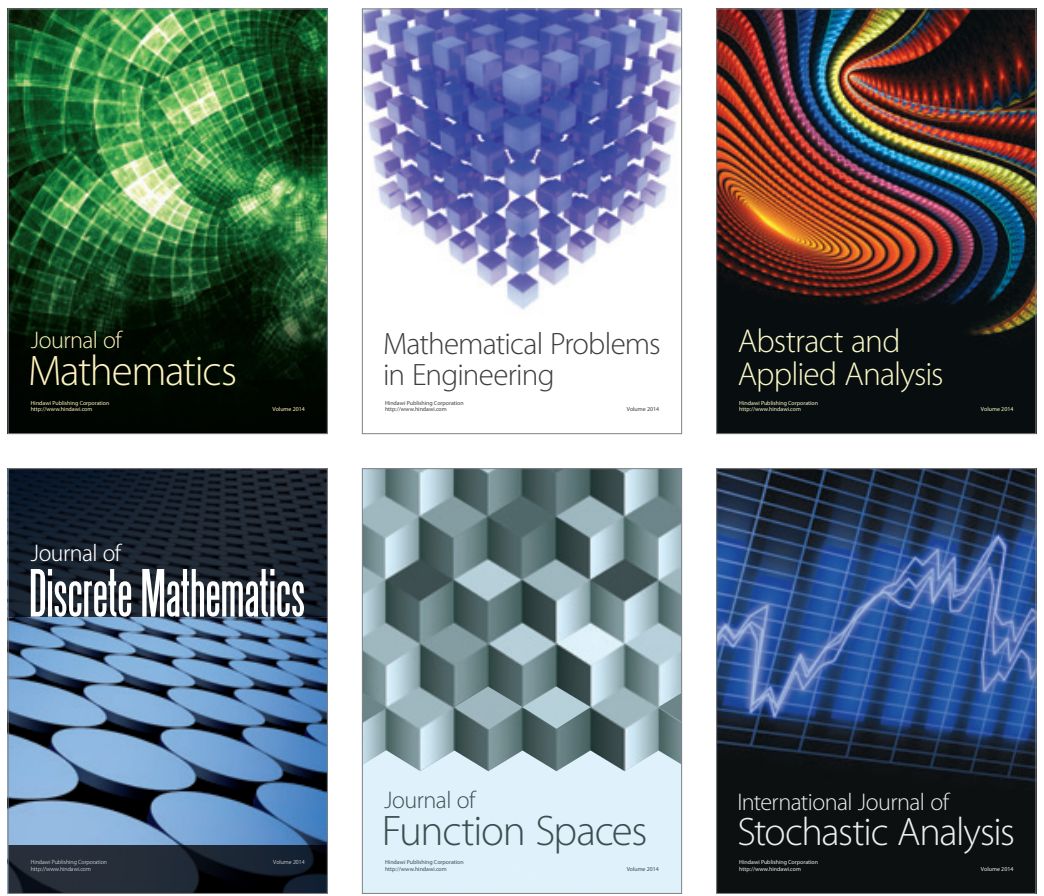

Journal of

Function Spaces

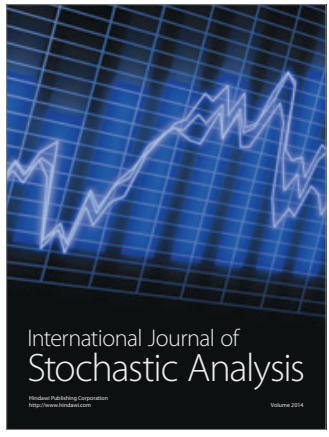

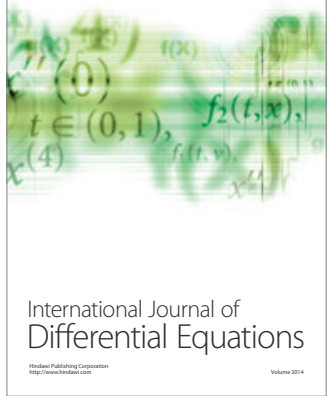
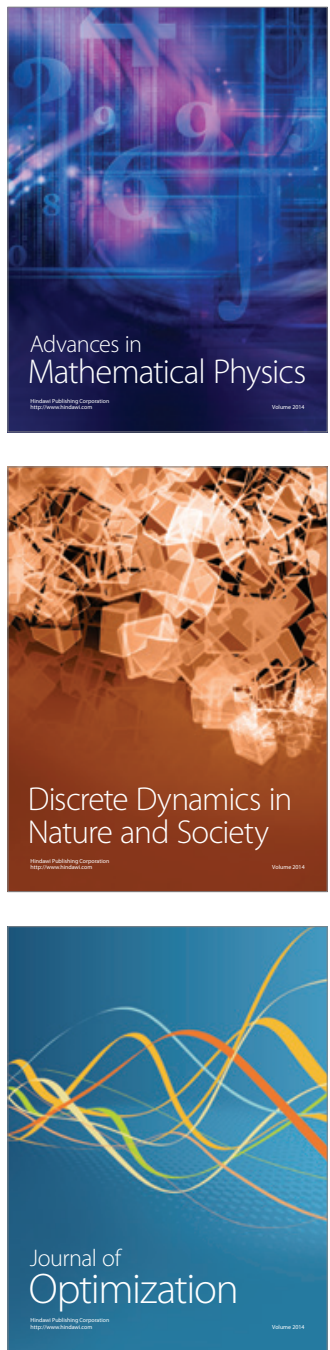